\title{
Investigation of MicroRNA in Mitochondrial Apoptotic Pathway in Systemic Lupus Erythematosus
}

\author{
Yu-Jih Su $\mathbb{D},{ }^{1}$ Nai-Wen Tsai, ${ }^{2}$ Chia-Te Kung, ${ }^{3}$ Hung-Chen Wang $\mathbb{D},{ }^{4}$ \\ Wei-Che Lin $\left(\mathbb{D},{ }^{5}\right.$ Chih-Cheng Huang $\left(\mathbb{D},{ }^{2}\right.$ Ya-Ting Chang $\left(\mathbb{D},{ }^{2}\right.$ Chih-Min Su, ${ }^{3}$ \\ Yi-Fang Chiang, ${ }^{2}$ Ben-Chung Cheng, ${ }^{6,7}$ Yu-Jun Lin, ${ }^{4,7}$ and Cheng-Hsien Lu $\mathbb{D}^{2,7,8}$ \\ ${ }^{1}$ Department of Rheumatology, Allergy and Immunology, Kaohsiung Chang Gung Memorial Hospital and Chang Gung University, \\ College of Medicine, Kaohsiung, Taiwan \\ ${ }^{2}$ Department of Neurology, Kaohsiung Chang Gung Memorial Hospital and Chang Gung University, College of Medicine, \\ Kaohsiung, Taiwan \\ ${ }^{3}$ Department of Emergency Medicine, Kaohsiung Chang Gung Memorial Hospital and Chang Gung University, College of Medicine, \\ Kaohsiung, Taiwan \\ ${ }^{4}$ Department of Neurosurgery, Kaohsiung Chang Gung Memorial Hospital and Chang Gung University, College of Medicine, \\ Kaohsiung, Taiwan \\ ${ }^{5}$ Department of Radiology, Kaohsiung Chang Gung Memorial Hospital and Chang Gung University, College of Medicine, \\ Kaohsiung, Taiwan \\ ${ }^{6}$ Department of Nephrology, Kaohsiung Chang Gung Memorial Hospital and Chang Gung University, College of Medicine, \\ Kaohsiung, Taiwan \\ ${ }^{7}$ Department of Biological Science, National Sun Yat-Sen University, Kaohsiung, Taiwan \\ ${ }^{8}$ Department of Neurology, Xiamen Chang Gung Memorial Hospital, Xiamen, China
}

Correspondence should be addressed to Cheng-Hsien Lu; chlu99@ms44.url.com.tw

Received 28 October 2017; Accepted 4 March 2018; Published 10 July 2018

Academic Editor: Ewa Mojs

Copyright (C) 2018 Yu-Jih Su et al. This is an open access article distributed under the Creative Commons Attribution License, which permits unrestricted use, distribution, and reproduction in any medium, provided the original work is properly cited.

Background. Accumulating evidence indicates that microRNAs play a pivotal role in the pathogenesis of systemic lupus erythematosus (SLE). This study tested the hypothesis that microRNA is associated with the mitochondrial apoptotic pathway in patients with SLE. Methods. Thirteen patients were in the clinical comparison study and microRNA study and overall 19 patients in the study of intracellular protein. Levels of microRNAs were determined by miRNeasy kit in 13 patients with SLE and 29 volunteer normal controls. Intracellular levels of caspase-9, caspase-10, MAVS, MDA5, and pIRF7 in mononuclear cells from 19 patiens and the SLE disease activity index (SLEDAI) were determined in all SLE patients. Correlation analyses were performed among microRNAs, intracellular adaptor proteins, and caspase levels and mean SLEDAI. Results. The $\triangle \mathrm{CT}$, defined by test reading difference between the target and the internal control microRNA (miR-451a), of miR-21-5p, miR-150-5p, and miR221-3p were significantly higher in plasma from SLE patients than in normal controls. miR-150-5p $\Delta$ CT was positively correlated with both CRP and SLEDAI value. miR-150-5p $\Delta$ CT was negatively associated with MAVS $70 \mathrm{kD}$. Caspase-10 protein levels were negatively associated with plasma miR-22-3p $\Delta \mathrm{CT}$ and miR21-5p $\Delta \mathrm{CT}$ levels. Conclusions. Our study confirmed the hypothesis that these microRNAs were associated with the mitochondrial apoptotic pathway in SLE. miR-150-5p $\Delta$ CT was positively associated with SLE disease activity and it was negatively correlated with MAVS $70 \mathrm{kD}$, which may facilitate viral survival and further enhance inflammation. On the other hand, miR-22-3p $\Delta \mathrm{CT}$ and miR-21-5p $\Delta$ CT, were negatively correlated with caspase-10 levels, which may repress extrinsic apoptosis and increase cell survival.

\section{Introduction}

Systemic lupus erythematosus (SLE) is a chronic systemic disease affecting mostly women of child-bearing age. It is the prototype of autoimmune diseases because of the variety of its proposed pathogenesis mechanisms. Chronic or acute viral infection or reactivation is one of several important mechanisms involved in the pathogenesis of this 
condition [1-6]. Few markers reflect antiviral immunity clinically, with the exception of the antiviral immunoglobulins (e.g., IgG, IgA, or IgM). The peripheral blood mononuclear cells, PBMCs, include both lymphocytes and monocytes by definition. In SLE patients, these two leukocyte lineages are key players in disease pathogenesis and are key cells that fight viral infection. The major functions of these two leukocyte lines are antigen presentation and the execution of adaptive immunity and interferon production against infection $[7,8]$.

Aside from mononuclear cells of leukocytes, viruses play a role in inducing lupus and lupus flare-ups [4, 9-11]. In addition to the incorporation of the interferon pathway, we focused on antiviral molecules such as mitochondrial antiviral signaling protein (MAVS), melanoma differentiationassociated protein 5 (MDA5), and interferon regulatory factor 7 (IRF7) in this study. The postviral immune response should activate IRF genes [12]. Changes in IRF7 phosphorylation levels could be explained by aberrant activation of the NLRP3 pathway [13], STAT1 pathways [14], IRF3 [15], or downstream MAVS signaling due to inflammation. On the other hand, it might be caused by autoimmunity or cytokine milieu in SLE [16-18].

Levels of plasma microRNAs are deliberately controlled, requiring multiple layers of regulation involving the participation of various protein regulators and posttranscriptional modifications [19-23]. This study explored the associations between circulating microRNA and intracellular proteins involved in the mitochondrial apoptotic pathway including caspase, pIRF7, MAVS, and MDA5. Because of the possible benefits of choosing the appropriate immunosuppressant regimen, there is a need to improve our understanding of the clinical significance of antiviral immunity in SLE.

\section{Patients and Methods}

2.1. Study Patients. The patients with definitive diagnosis of SLE who were followed up at the Rheumatology Outpatient Clinic for more than six months were prospectively evaluated and compared to 29 healthy subjects. The diagnostic of SLE was based on the 1997 revision of the 1982 American College of Rheumatology classification criteria for SLE [24], and the assessment of SLE disease activity was based on the SLE disease activity index (SLEDAI) [25].

There were 19 SLE patients enrolled, and all patients did not undergo changes in steroid dose or immune-modifying medication during the study period. For comparison, 29 ageand sex-matched healthy subjects were enrolled as healthy controls. The individual plasma microRNA was retrieved in 13 SLE subjects, but the experiment from the rest of six SLE patients was suboptimal. In total, there were 13 patients accomplished in the plasma microRNA and clinical comparison study and 19 patients in the study of intracellular protein study.

The Institutional Review Committee on Human Research reviewed and approved the study protocol and all participants provided informed consent. Patients were excluded if they had autoimmune diseases other than SLE.
2.2. Clinical Assessments. All 19 subjects had complete medical examinations upon enrollment. Clinical data including complement levels and anti-double strand DNA levels were performed regularly and collected upon enrollment. Biomarkers, including demography data, complement levels, anti-ribosomal p autoantibody (a-rib p), anti-double strand DNA autoantibody (a-dsDNA) levels, and disease activity index were also collected.

\subsection{Assessment of Protein Expression and MicroRNA Levels}

2.3.1. Western Blot Analysis. Levels of intracellular proteins, including MAVS (57 kD and $70 \mathrm{kD})$, pIRF7 (65 kD), caspase$9(37 \mathrm{kD})$, caspase-10 $(59 \mathrm{kD})$, and MDA5 $(135 \mathrm{kD})$, were determined by western blotting. The MAVS were defined as the larger one $(70 \mathrm{kD})$ and the smaller one, mini-MAVS (57 kD) [26-29]. Blood samples were collected by venipuncture of forearm veins of the 19 SLE patients. Peripheral blood mononuclear cell (PBMC) intracellular protein levels of phosphorylated interferon regulator factor 7 (pIRF7), mitochondrial antiviral signaling protein (MAVS), and melanoma differentiation-associated protein 5 (MDA5) were detected by western blotting. Detailed procedures were described in the previous study [30]. The reagents and antibodies were rabbit polyclonal antibodies recognizing caspase-9 (Cell Signaling, \#9501), phospho-IRF-7 (Cell signaling, \#5184), rat polyclonal antibodies recognizing caspase-10 (Biolegend, \#645202), and anti-mitochondrial antiviral signaling antibody (MAVS) (Abcam \#ab25084).

Caspase- 9 activation was demonstrated by observing cleaved caspase- 9 (active caspase-9, caspase-9c, $37 \mathrm{kD}$ ) from original caspase-9 (caspase-9, $47 \mathrm{kD}$ ) [18]. Caspase-10 activation was demonstrated by observing cleaved caspase-10 (active caspase-10, caspase-10c, $43 \mathrm{kD}$ ) from original caspase10 (caspase-10, 59kD) [19]. The MAVS were shown to have two types with similar activities: $70 \mathrm{kD}$ (full-length MAVS) and $57 \mathrm{kD}$ (mini-MAVS) [27, 29, 31, 32].

2.3.2. MicroRNA Measurement. Blood samples were collected by venipuncture of forearm veins of the 19 SLE patients and 29 normal subjects. Samples were centrifuged at $1000 \times g$ for $10 \mathrm{~min}$ to pellet cellular debris. The supernatant was used for RNA extraction. Total RNA was extracted from $300 \mu \mathrm{L}$ of fluid using the miRNeasy kit (Qiagen) as described by Weber et al. [33]. Amplification was performed according to the manufacturer's instructions (Qiagen). We assessed the extracted RNA for quality and quantity using an Agilent 2100 Bioanalyzer and NanoDrop 1000 spectrophotometer (Thermo Scientific). For the bioanalyzer, the RNA 6000 Pico chip was used for quantification and an initial quality measurement, followed by the use of a Small RNA chip to gain a more detailed view of RNAs in the 6- to 150-nucleotide size range. We performed quantitative real-time PCR (qPCR) according to manufacturer's instructions (Qiagen) to profile the miRNA distribution in body fluid samples. In brief, $5 \mu \mathrm{L}$ total RNA was collected and pooled from the samples of the same fluid type, and the cDNA was produced using the miScript Reverse Transcription kit (Qiagen). We used the Matrix Hydra eDrop (Thermo Scientific) to mix the cDNA 
sample and the qPCR master reagent [Human miScript Assay 384 set v10.1 (Qiagen)] to reduce pipetting error. Any wells with multiple melting temperature values were excluded from further analysis. We also used individual Human miScript Assays to validate the 384 miRNA qPCR set. Data was analyzed using SDS Enterprise Database 2.3 (Applied Biosystems) and normalized to a global mean instead of specific miRNA or noncoding RNA signals.

2.4. Statistical Analysis. Data were expressed in the form of mean $\pm \mathrm{SD}$ or median (interquartile range). Categorical variables were compared by Chi-square test or Fisher's exact test. Continuous variables were arcsine-transformed to improve normality, and then comparisons between two groups were performed using Student's $t$-test. Correlation analysis was used to explore the relationship between the SLEDAI score and variables such as microRNA and intracellular protein levels. Spearman's rho for nonlinear distributed variables and Pearson correlation were used for linear distributed variables. The statistical significance threshold was set at $p<0.05$. All statistical calculations were performed by using the SAS software package, version 9.1 (2002, SAS Statistical Institute, North Carolina).

\section{Results}

3.1. Baseline Characteristics of the Study Patients. The baseline characteristics, laboratory data, and microRNA of the SLE patients and healthy controls are listed in Table 1 . The age and gender distributions were similar between SLE and normal controls ( $p=0.07$ and 0.37 , respectively). The disease activity (SLEDAI-2k) of the 13 lupus patients was $6.08 \pm 4.87$, with the highest at 17 and lowest at 2. The clinical symptoms of the 13 SLE patients included neurologic involvement in three patients, musculoskeletal involvement in ten patients, hematologic involvement in three patients, renal involvement in one patient, cardiac involvement in one patient, respiratory involvement in two patients, and mucocutaneous involvement in two patients. Six SLE patients had involvement of more than one organ. Overall, these SLE patients were under medication control in a relatively stable disease condition who were regularly followed up with at outpatient clinics. The leukocyte, hemoglobin, c-reactive protein (CRP), liver enzymes, and creatinine levels were similar between the two groups (all $p>0.05$ ), which demonstrated the stable and steady state of the SLE patients. The only difference between the two groups was the total cholesterol and the triglyceride levels, which were significantly higher in SLE patients than in normal controls (both $p<0.05$ ), but this conferred no clinical significance (comparable statin usage between the two groups, $p=0.64$ ) (Table 1).

3.2. MicroRNAs Expression in Patients with SLE. The levels of plasma microRNAs were significantly lower in three out of four microRNAs selected in this study (Table 1). Among them, the $\Delta C T$ of miR-21-5p, miR-150-5p, and miR221-3p were significantly higher in plasma from SLE patients than in normal controls (all $p<0.05$, higher $\Delta \mathrm{CT}$ indicates lower plasma level), except miR-22-3p. The levels of miR-22-3p were similar between SLE patients and normal controls $(p>$ 0.05).

3.3. Correlations Analysis between MicroRNA and Leukocyte Viral Infection/Activation Markers. The association between microRNA and the intracellular protein levels including caspase-9, caspase-10, MDA5, full-length MAVS (70 kD), and mini-MAVS $(57 \mathrm{kD})$, is listed in Table 2, and the western blot data is shown in Figure 1. The $\Delta$ CT of miR-150-5p was positively correlated with SLEDAI $(r=0.63, p=0.01)$. The $\triangle \mathrm{CT}$ of miR-150-5p was also negatively associated with fulllength MAVS level ( $r=-0.49, p=0.04$, Table 2$)$. Caspase-10 protein levels were negatively associated with plasma miR-22$3 \mathrm{p}(r=-0.47, p<0.05)$ and miR-21-5p $(r=-0.62, p=$ $0.01)$. Further, the $\Delta \mathrm{CT}$ of $\mathrm{miR}-150-5 \mathrm{p}$ was positively correlated with $\operatorname{CRP}(r=0.56, p<0.01)$.

\section{Discussion}

The present study examined the role of microRNA in mitochondrial apoptotic pathway in SLE, with several major findings. First, the $\Delta$ CT of miR-21-5p, miR-150-5p, and miR221-3p were significantly higher in plasma from SLE patients than in normal controls (all $p<0.05$ ) (Table 1). Second, the $\triangle \mathrm{CT}$ of miR-150-5p was positively correlated with both the SLEDAI and CRP. Third, the $\triangle \mathrm{CT}$ of miR-150-5p is negatively associated with active MAVS and $\Delta \mathrm{CT}$ of miR-22-3p $(r=$ $-0.47, p<0.05)$ and $\Delta$ CT of miR-21-5p $(r=-0.62, p=0.01)$ were negatively associated with caspase-10 protein levels.

There is accumulating evidence about the role of microRNA in the pathogenesis of SLE and autoimmune diseases. For example, mir-150 regulates various immune cells, including B cells, T cells, and NK cells [34], and it is a biomarker in lupus nephritis [35], but its expression does not differ in $\mathrm{T}$ cells from lupus patients and normal controls [21]. On the other hand, this miRNA is downregulated in skin with psoriasis [20] but increased in plasma during osteogenesis [36] and scleroderma [34] or after a 10-km race [37]. These results suggest that microRNA expression and effects could be tissue-specific and that plasma microRNA reflects a general condition of a patient.

This study linked mir-150-5p with intracellular MAVS protein expression. The MAVS protein levels were significantly higher in patients with lupus than in normal controls and were negatively correlated with lupus activity in our previous study [30]. The dynamic changes of mir-150-5p relating to lupus activity are worthy of further investigation.

Cell catabolism is upregulated in SLE, and several recent articles have mentioned apoptosis and microRNAs in SLE $[19,20,23,38]$. Wang et al. showed that miR221/222 downregulates caspase-10 in vitro [23]. As seen in Table 2, we noted that caspase-10 is negatively associated with $\Delta \mathrm{CT}$ of several plasma microRNAs, including miR-22-3p and miR21-5p. This observation suggests the activation of the extrinsic apoptotic caspase-10-related pathway in SLE [22, 39] and that caspase-10 is influenced by microRNA, which could be reflected by plasma microRNA levels in this study. Furthermore, we previously demonstrated that caspase-10 positively correlates with pIRF7, and caspase- 9 and caspase-10 both 


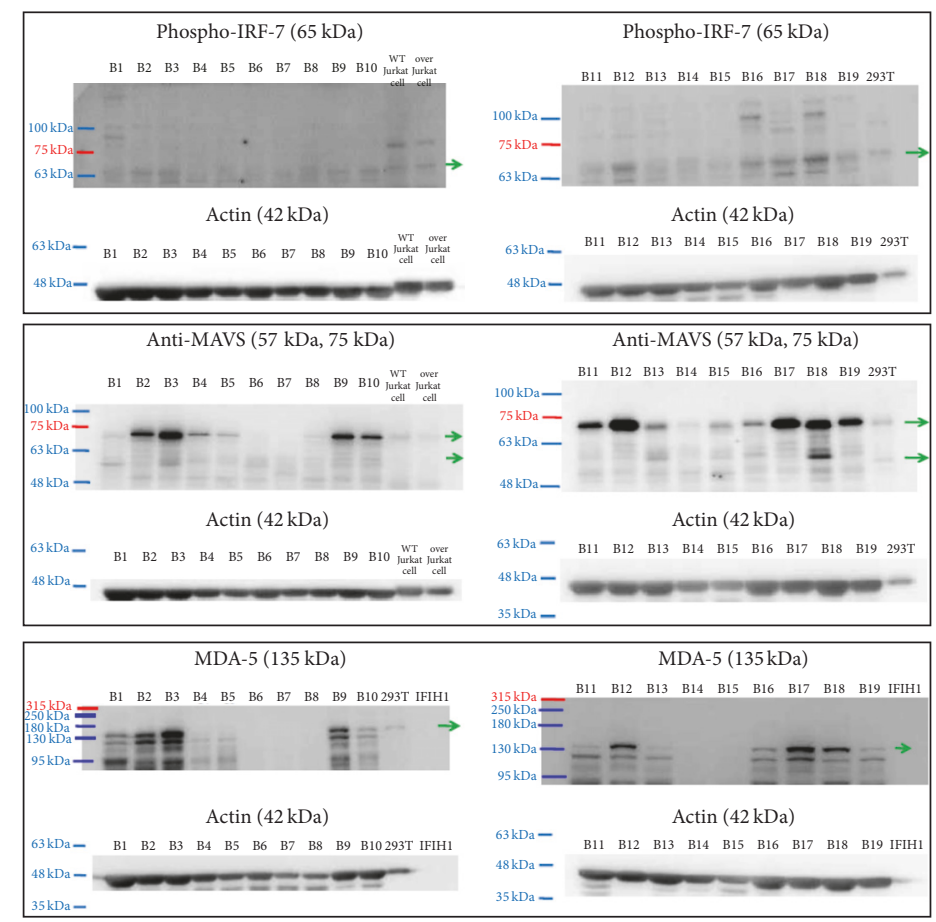

(a)

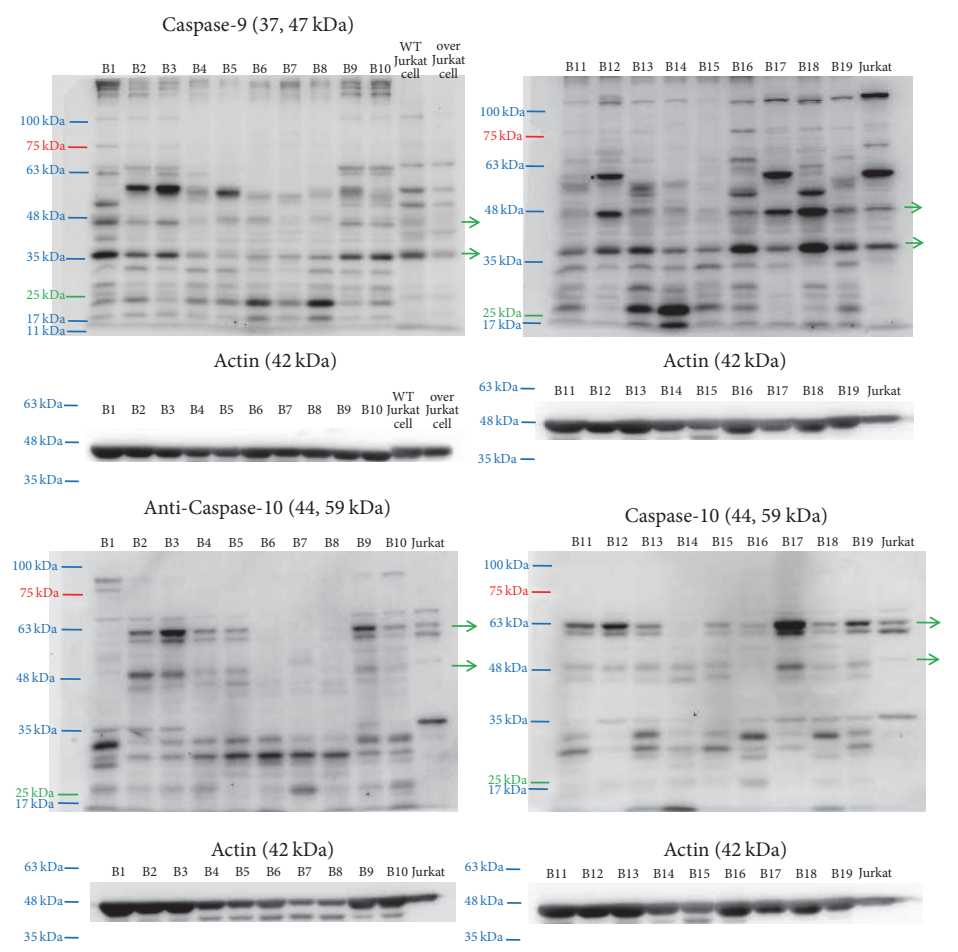

(b)

FIGURE 1: Protein expression in peripheral blood mononuclear cells from systemic lupus erythematosus patients. (a) Intracellular proteins (pIRF7, MAVS, and MDA5) in western blots. (b) Intracellular proteins (caspase-9 and caspase-10) in western blots. pIRF7, phosphorylated interferon regulator factor 7; MAVS, mitochondrial antiviral signaling protein; MDA5, melanoma differentiation-associated protein 5; B1 B19, peripheral mononuclear cell lysate from systemic lupus erythematosus patients. 
TABLE 1: Demographic clinical data of SLE patients and healthy controls.

\begin{tabular}{|c|c|c|c|}
\hline & $\begin{array}{l}\text { Normal controls } \\
\qquad(n=29)\end{array}$ & $\begin{array}{c}\text { SLE } \\
(n=13)\end{array}$ & $p$ value \\
\hline Age (year) & $57.76 \pm 5.47$ & $51.31 \pm 11.10$ & 0.07 \\
\hline Leukocytes $(\times 1000 / \mathrm{ml})$ & $5.53 \pm 1.40$ & $6.68 \pm 2.24$ & 0.11 \\
\hline Hemoglobin (mg/dL) & $13.37 \pm 1.66$ & $12.44 \pm 1.74$ & 0.12 \\
\hline Hematocrit (\%) & $40.57 \pm 3.75$ & $37.40 \pm 4.71$ & 0.05 \\
\hline c-reactive protein $(\mathrm{mg} / \mathrm{dL})$ & $1.75 \pm 2.29$ & $5.98 \pm 7.54$ & 0.23 \\
\hline Aspartate aminotransferase $(\mathrm{U} / \mathrm{dL})$ & $23.88 \pm 7.63$ & $40.88 \pm 56.02$ & 0.42 \\
\hline Alanine aminotransferase (U/dL) & $24.65 \pm 17.12$ & $19.13 \pm 8.36$ & 0.39 \\
\hline Total cholesterol (mg/dL) & $196.9 \pm 26.89$ & $221.57 \pm 34.07$ & $<0.05^{*}$ \\
\hline high-density lipoprotein (mg/dL) & $59.86 \pm 14.85$ & $67.17 \pm 19.41$ & 0.31 \\
\hline low-density lipoprotein (mg/dL) & $118.62 \pm 23.05$ & $117.83 \pm 26.23$ & 0.94 \\
\hline Triglyceride (mg/dL) & $102.83 \pm 52.50$ & $191.71 \pm 86.69$ & $0.04^{*}$ \\
\hline Creatinine $(\mathrm{mg} / \mathrm{dL})$ & $0.71 \pm 0.15$ & $0.86 \pm 0.49$ & 0.37 \\
\hline Gender (female: male) & 21:08 & 11:02 & 0.47 \\
\hline Use of statins (yes: no) & $03: 26$ & 01:12 & 0.64 \\
\hline \multicolumn{4}{|c|}{ plasma microRNA: miR-54la as control (CT) } \\
\hline miR-22-3p $\Delta \mathrm{CT}$ & $6.24 \pm 1.51$ & $6.36 \pm 0.84$ & 0.79 \\
\hline miR-150-5p $\Delta C T$ & $7.07 \pm 1.60$ & $8.71 \pm 1.46$ & $<0.01^{*}$ \\
\hline miR-221-3p $\Delta \mathrm{CT}$ & $5.88 \pm 2.19$ & $7.65 \pm 1.56$ & $0.01^{*}$ \\
\hline miR-21-5p $\Delta \mathrm{CT}$ & $2.55 \pm 1.43$ & $4.10 \pm 0.92$ & $<0.01^{*}$ \\
\hline
\end{tabular}

SLE, systemic lupus erythematosus; $\S$, data presented with mean \pm SD (standard deviation); continuous variables between two groups were compared using Student's $T$-test, between $\alpha$, healthy group, and $\beta$, SLE; Gender and use of statins were compared using Fisher's exact test; $*$ indicates $p$ value $<0.05$.

TABLE 2: Correlation analysis between microRNA and leukocyte viral infection/activation markers.

\begin{tabular}{|c|c|c|c|c|c|c|c|}
\hline$n=13$ & SLEDAI & casp-9 $(37 \mathrm{kD})$ & casp-10 (59 kD) & MDA5 & MAVS (70 kD) & MAVS (57 kD) & CRP \\
\hline \multicolumn{8}{|c|}{$\begin{array}{l}\text { miR-22-3p } \\
\Delta \mathrm{CT}\end{array}$} \\
\hline$r$ & -.12 & -.08 & $-.47^{*}$ & .08 & .10 & -.38 & -.09 \\
\hline$p$ & .63 & .75 & $<.05^{*}$ & .77 & .69 & .13 & .65 \\
\hline \multicolumn{8}{|c|}{$\begin{array}{l}\text { miR-150-5p } \\
\Delta \mathrm{CT}\end{array}$} \\
\hline$r$ & $.70^{*}$ & -.03 & -.13 & -.36 & $-.49^{*}$ & -.06 & $.56^{*}$ \\
\hline$p$ & $.00^{*}$ & .91 & .60 & .15 & $.04^{*}$ & .81 & $<.01$ \\
\hline \multicolumn{8}{|c|}{$\begin{array}{l}\text { miR-221-3p } \\
\Delta \mathrm{CT}\end{array}$} \\
\hline$r$ & -.12 & -.12 & -.02 & .13 & .21 & -.39 & .09 \\
\hline$p$ & .65 & .64 & .93 & .61 & .40 & .11 & .65 \\
\hline \multicolumn{8}{|c|}{$\begin{array}{l}\text { miR-21-5p } \\
\Delta \mathrm{CT}\end{array}$} \\
\hline$r$ & .02 & -.23 & $-.62^{*}$ & .01 & -.07 & -.21 & .23 \\
\hline$p$ & .92 & .35 & $.01^{*}$ & .98 & .78 & .40 & .21 \\
\hline
\end{tabular}

Method: Spearman's rho for nonlinear distributed variables or Pearson correlation for linear distributed variables; $r$, correlation coefficient; $p, p$ value; $n$, number; casp, caspase; SLEDAI-2K, systemic lupus erythematosus disease activity index 2000; $\triangle \mathrm{CT}$, compared with miR-541a as control (CT); pIRF7, phosphorylated interferon regulator factor 7; MAVS, mitochondrial antiviral signaling protein; MDA5, melanoma differentiation-associated protein 5; CRP, c-reactive protein $(\mathrm{mg} / \mathrm{dL}) ; *$, correlation is significant at the 0.05 level.

positively correlate with each other, pIRF7, and MAVS $70 \mathrm{kD}$ [30], indicating that interferons are links to cellular apoptosis and anti-virus immunity in SLE [16, 40-42]. The pathways of MAVS and caspase-10 were examined by detecting plasma microRNA in SLE (Figure 2).
A new direction of SLE studies in microRNA shed light on SLE pathogenesis [43]. Our preliminary study adds several microRNAs that were significantly different from normal controls to the list of previously identified microRNAs. It is worth mentioning that miR-150-5p levels were positively 


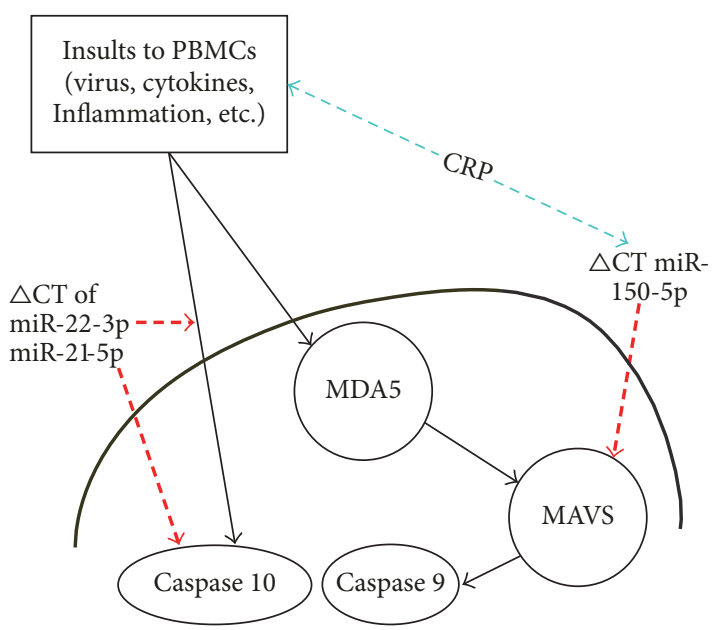

FIGURE 2: The simplified link between $\Delta \mathrm{CT}$ of each microRNA and apoptotic molecules in peripheral blood mononuclear cells (PBMCs) in our study. Bidirection (blue) arrow-head thin dash-line: positive correlation between $\triangle \mathrm{CT}$ microRNA and serum protein; arrow-head (red) bold dash-line: negative correlation between $\triangle \mathrm{CT}$ microRNA and intracellular protein levels. CRP, C-reactive protein; MAVS, mitochondrial antiviral signaling protein; MDA5, melanoma differentiation-associated protein 5; PBMCs, peripheral blood mononuclear cells.

correlated with clinical inflammatory indicator CRP level and the SLEDAI but were negatively associated with MAVS. The idea that interplay between disease activity and infectious disease could be linked by microRNA has been mentioned in other studies $[34,44]$.

This study had several limitations. First, this was a cross-sectional observational study. More detailed studies are required to determine the real function of plasma microRNA levels in lupus. The concentration of plasma microRNA is low and it needs delicate handling during experiment procedure, and six of our patients' plasma microRNAs were either undetectable or with poor quality which prevent further analysis. A longitudinal study is also required to detect the trend of plasma microRNA in lupus and could reduce variance and improve our ability to predict the prognoses. Second, the case number in this study was small. The difference data number between the plasma microRNA and the intracellular protein levels was due to experiment difficulty of the retrieving plasma microRNA. Large-scale prospective and longitudinal studies are needed to evaluate the prognostic contribution of microRNAs on clinical outcome.

Our study confirmed the hypothesis that these microRNAs were associated with the mitochondrial apoptotic pathway in SLE. MiR-150-5p $\Delta$ CT was positively associated with SLE disease activity and was negatively correlated with MAVS $70 \mathrm{kD}$. The level of microRNA concentration is reversed to the $\Delta \mathrm{CT}$, so it is suggesting that this miR-150-5p is positively correlated with MAVS $70 \mathrm{kD}$ and might facilitate anti-viral activity during viral infection and this might be reflected by elevation of CRP levels clinically. The miR-150-5p could be one useful marker demonstrating virus related lupus disease flare-up clinically (Figure 2). On the other hand, miR-22-3p
$\Delta \mathrm{CT}$ and miR-21-5p $\Delta \mathrm{CT}$ were negatively correlated with caspase-10 levels, where these microRNAs may associate increased extrinsic apoptosis and decreased cell survival, which could reflect monocyte activation-induced cell death.

In conclusion, the plasma microRNA could be a maker demonstrating complex immune milieu in lupus. Some specific microRNA markers could be useful makers for differentiating intracellular immune pathways, such as miR$150-5 p$ in MAVS pathway and miR-22-3p and miR-21-5p in extrinsic apoptosis pathway.

All the underlying research materials related to our article can be accessed on demand by email notification.

\section{Ethical Approval}

The study was approved by Chang Gung Memorial Hospital's Institutional Review Committee on Human Research.

\section{Conflicts of Interest}

The authors declare that they have no conflicts of interest.

\section{Authors' Contributions}

Yu-Jih Su participated in the design of the study and drafted the manuscript. Chia-Te Kung, Hung-Chen Wang, Wei-Che Lin, Chih-Cheng Huang, Ya-Ting Chang, Chih-Min Su, YiFang Chiang, Ben-Chung Cheng, and Yu-Jun Lin participated in the sequence alignment and clinical evaluation of patients. Nai-Wen Tsai performed the statistical analysis. Cheng-Hsien Lu conceived the study, participated in its design and coordination, and helped draft the manuscript. All authors read and approved the final manuscript.

\section{Acknowledgments}

This work was supported by grants from Chang Gung Memorial Hospital-Kaohsiung Medical Center (CMRPG8G0351). The authors would like to thank Drs. Tien-Tsai Cheng and Chung-Jen Chen for brainstorming.

\section{References}

[1] A. Migliorini and H.-J. Anders, "A novel pathogenetic conceptantiviral immunity in lupus nephritis," Nature Reviews Nephrology, vol. 8, no. 3, pp. 183-189, 2012.

[2] S. P. Crampton, J. A. Deane, L. Feigenbaum, and S. Bolland, "Ifihl gene dose effect reveals MDA5-mediated chronic type I IFN gene signature, viral resistance, and accelerated autoimmunity," The Journal of Immunology, vol. 188, no. 3, pp. 1451-1459, 2012.

[3] R. Higgs, E. Lazzari, C. Wynne et al., "Self protection from antiviral responses-Ro52 promotes degradation of the transcription factor IRF7 downstream of the viral Toll-like receptors," PLoS ONE, vol. 5, no. 7, Article ID el1776, 2010.

[4] B. Y. Su, C. Y. Su, S. F. Yu, and C. J. Chen, "Incidental discovery of high systemic lupus erythematosus disease activity associated with cytomegalovirus viral activity," Medical Microbiology and Immunology, vol. 196, pp. 165-170, 2007.

[5] P. S. Patole, H. J. Grone, S. Segerer et al., "Viral double-stranded RNA aggravates lupus nephritis through Toll-like receptor 3 on 
glomerular mesangial cells and antigen-presenting cells," Journal of the American Society of Nephrology: JASN, vol. 16, pp. 1326-1338, 2005.

[6] J. R. Yuste and J. Prieto, "Anticardiolipin antibodies in chronic viral hepatitis. Do they have clinical consequences?" European Journal of Gastroenterology \& Hepatology, vol. 15, no. 7, pp. 717719, 2003.

[7] X. Gong, J. Zhou, W. Zhu et al., "Excessive proinflammatory cytokine and chemokine responses of human monocytederived macrophages to enterovirus 71 infection," BMC Infectious Diseases, vol. 12, p. 224, 2012.

[8] P. Ekchariyawat, A. Thitithanyanont, S. Sirisinha, and P. Utaisincharoen, "Apoptosis induced by avian $\mathrm{H} 5 \mathrm{~N} 1$ virus in human monocyte-derived macrophages involves TRAIL-inducing caspase-10 activation," Journal of Innate Immunity, vol. 18, no. 3, pp. 390-397, 2012.

[9] B. R. Berner, M. Tary-Lehmann, N. L. Yonkers, A. D. Askari, P. V. Lehmann, and D. D. Anthony, "Phenotypic and functional analysis of EBV-specific memory CD8 cells in SLE," Cellular Immunology, vol. 235, no. 1, pp. 29-38, 2005.

[10] D. H. Dreyfus, A. J. Gross, and D. Thorley-Lawson, "Role of T Cells in EBV-Infected Systemic Lupus Erythematosus Patients," The Journal of Immunology, vol. 175, no. 6, pp. 3460-3461, 2005.

[11] A. J. Gross, D. Hochberg, W. M. Rand, and D. A. Thorley-Lawson, "EBV and systemic lupus erythematosus: A new perspective," The Journal of Immunology, vol. 174, no. 11, pp. 6599-6607, 2005.

[12] K. Honda, H. Yanai, H. Negishi et al., "IRF-7 is the master regulator of type-I interferon-dependent immune responses," Nature, vol. 434, no. 7034, pp. 772-777, 2005.

[13] S. Park, C. Juliana, S. Hong et al., “The mitochondrial antiviral protein MAVS associates with NLRP3 and regulates its inflammasome activity," The Journal of Immunology, vol. 191, no. 8, pp. 4358-4366, 2013.

[14] R. Nandakumar, K. Finsterbusch, C. Lipps et al., "Hepatitis C virus replication in mouse cells is restricted by IFN-dependent and -independent mechanisms," Gastroenterology, vol. 145, no. 6, pp. 1414-1423.el, 2013.

[15] N. C. Di Paolo, K. Doronin, L. K. Baldwin, T. Papayannopoulou, and D. M. Shayakhmetov, "The transcription factor IRF3 triggers defensive suicide necrosis in response to viral and bacterial pathogens," Cell Reports, vol. 3, pp. 1840-1846, 2013.

[16] Q. Fu, J. Zhao, X. Qian et al., "Association of a functional IRF7 variant with systemic lupus erythematosus," Arthritis \& Rheumatology, vol. 63, no. 3, pp. 749-754, 2011.

[17] F. Wang, Y. F. Zou, G. P. Sun et al., "Association between functional IRF7 variant and systemic lupus erythematosus may need more critical examination: comment on the article by $\mathrm{Fu}$ et al," Arthritis and Rheumatism, vol. 63, pp. 3177-3178, 2011.

[18] S. Uematsu, S. Sato, M. Yamamoto et al., "Interleukin-1 receptorassociated kinase-1 plays an essential role for Toll-like receptor (TLR)7- and TLR9-mediated interferon- $\alpha$ induction," The Journal of Experimental Medicine, vol. 201, no. 6, pp. 915-923, 2005.

[19] A. L. Carlsen, A. J. Schetter, C. T. Nielsen et al., "Circulating microRNA expression profiles associated with systemic lupus erythematosus," Arthritis \& Rheumatology, vol. 65, no. 5, pp. 1324-1334, 2013.

[20] Y. Ding, W. Liao, Z. Yi, W. Xiang, and X. He, "Association of miRNA-145 expression in vascular smooth muscle cells with vascular damages in patients with lupus nephritis," International Journal of Clinical and Experimental Pathology, vol. 8, no. 10, pp. 12646-12656, 2015.
[21] M. C. Lu, N. S. Lai, H. C. Chen et al., "Decreased microRNA(miR)-145 and increased miR-224 expression in T cells from patients with systemic lupus erythematosus involved in lupus immunopathogenesis," Clinical and Experimental Immunology, vol. 171, pp. 91-99, 2013.

[22] M. Rastin, M. R. Hatef, N. Tabasi, and M. Mahmoudi, "The pathway of estradiol-induced apoptosis in patients with systemic lupus erythematosus," Clinical Rheumatology, vol. 31, no. 3, pp. 417-424, 2012.

[23] L. Wang, C. Liu, C. Li et al., "Effects of microRNA-221/222 on cell proliferation and apoptosis in prostate cancer cells," Gene, vol. 572, no. 2, pp. 252-258, 2015.

[24] E. L. Smith and R. H. Shmerling, "The American College of Rheumatology criteria for the classification of systemic lupus erythematosus: strengths, weaknesses, and opportunities for improvement," Lupus, vol. 8, no. 8, pp. 586-595, 1999.

[25] C. Bombardier, D. D. Gladman, M. B. Urowitz, D. Caron, and C. H. Chang, "Derivation of the SLEDAI. A disease activity index for lupus patients. The Committee on Prognosis Studies in SLE," Arthritis and Rheumatism, vol. 35, pp. 630-640, 1992.

[26] L. Qu, Z. Feng, D. Yamane et al., "Disruption of tlr3 signaling due to cleavage of trif by the hepatitis a virus protease-polymerase processing intermediate, $3 \mathrm{~cd}$," PLoS Pathogens, vol. 7, no. 9, Article ID e1002169, 2011.

[27] C. Wei, C. Ni, T. Song et al., "The hepatitis B virus X protein disrupts innate immunity by downregulating mitochondrial antiviral signaling protein," The Journal of Immunology, vol. 185, no. 2, pp. 1158-1168, 2010.

[28] H. Oshiumi, M. Ikeda, M. Matsumoto et al., "Hepatitis C virus core protein abrogates the DDX3 function that enhances IPS-1mediated IFN-beta induction," PLoS ONE, vol. 5, no. 12, Article ID e14258, 2010.

[29] X. D. Li, L. Sun, R. B. Seth, G. Pineda, and Z. J. Chen, “Hepatitis $\mathrm{C}$ virus protease NS3/4A cleaves mitochondrial antiviral signaling protein off the mitochondria to evade innate immunity," Proceedings of the National Academy of Sciences of the United States of America, vol. 102, pp. 17717-17722, 2005.

[30] Y. J. Su, T. T. Cheng, C. J. Chen et al., "Investigation of the caspase-dependent mitochondrial apoptotic pathway in mononuclear cells of patients with systemic lupus erythematosus," Journal of Translational Medicine, vol. 12, p. 303, 2014.

[31] Y. Yang, Y. Liang, L. Qu et al., "Disruption of innate immunity due to mitochondrial targeting of a picornaviral protease precursor," Proceedings of the National Acadamy of Sciences of the United States of America, vol. 104, no. 17, pp. 7253-7258, 2007.

[32] C. Buss, B. Opitz, A. C. Hocke et al., "Essential role of mitochondrial antiviral signaling, IFN regulatory factor (IRF)3, and IRF7 in Chlamydophila pneumoniae-mediated IFN-beta response and control of bacterial replication in human endothelial cells," The Journal of Immunology, vol. 184, pp. 3072-3078, 2010.

[33] J. A. Weber, D. H. Baxter, S. Zhang et al., "The microRNA spectrum in 12 body fluids," Clinical Chemistry, vol. 56, no. 11, pp. 1733-1741, 2010.

[34] X.-L. Huang, L. Zhang, J.-P. Li, Y.-J. Wang, Y. Duan, and J. Wang, "MicroRNA-150: A potential regulator in pathogens infection and autoimmune diseases," Autoimmunity, vol. 48, no. 8, pp. 503-510, 2015.

[35] H. Zhou, S. A. Hasni, P. Perez et al., "MiR-150 promotes renal fibrosis in lupus nephritis by downregulating SOCS1," Journal of the American Society of Nephrology, vol. 24, no. 7, pp. 10731087, 2013. 
[36] Y. Tian, J. Liu, X. Bai et al., "MicroRNA expression profile of surgical removed mandibular bone tissues from patients with mandibular prognathism," Journal of Surgical Research, vol. 198, no. 1, pp. 127-134, 2015.

[37] D. de Gonzalo-Calvo, A. Davalos, A. Montero et al., "Circulating inflammatory miRNA signature in response to different doses of aerobic exercise," Journal of Applied Physiology, vol. 119, pp. 124-134, 2015.

[38] M. C. Lu, C. L. Yu, H. C. Chen, H. C. Yu, H. B. Huang, and N. S. Lai, "Aberrant $\mathrm{T}$ cell expression of $\mathrm{Ca} 2+$ influx-regulated miRNAs in patients with systemic lupus erythematosus promotes lupus pathogenesis," Rheumatology, vol. 54, pp. 343-348, 2015.

[39] D. Mevorach, "Systemic lupus erythematosus and apoptosis: A question of balance," Clinical Reviews in Allergy \& Immunology, vol. 25, no. 1, pp. 49-60, 2003.

[40] D. Feng and B. J. Barnes, "Bioinformatics analysis of the factors controlling type I IFN gene expression in autoimmune disease and virus-induced immunity," Frontiers in Immunology, vol. 4, p. 291, 2013.

[41] R. M. Valente, E. Ehlers, D. Xu et al., “Toll-Like Receptor 7 Stimulates the Expression of Epstein-Barr Virus Latent Membrane Protein 1," PLoS ONE, vol. 7, no. 8, Article ID e43317, 2012.

[42] R. Salloum and T. B. Niewold, "Interferon regulatory factors in human lupus pathogenesis," Translational Research: the Journal of Laboratory and Clinical Medicine, vol. 157, pp. 326-331, 2011.

[43] D. Liang and N. Shen, "MicroRNA involvement in lupus: The beginning of a new tale," Current Opinion in Rheumatology, vol. 24, no. 5, pp. 489-498, 2012.

[44] E. N. Voynova, J. Skinner, and S. Bolland, "Expansion of an atypical NK cell subset in mouse models of systemic lupus erythematosus," The Journal of Immunology, vol. 194, no. 4, pp. 15031513, 2015. 


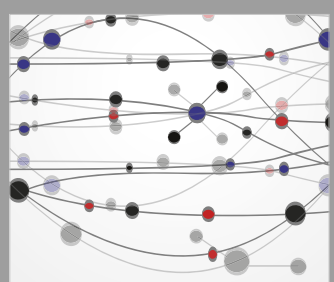

The Scientific World Journal
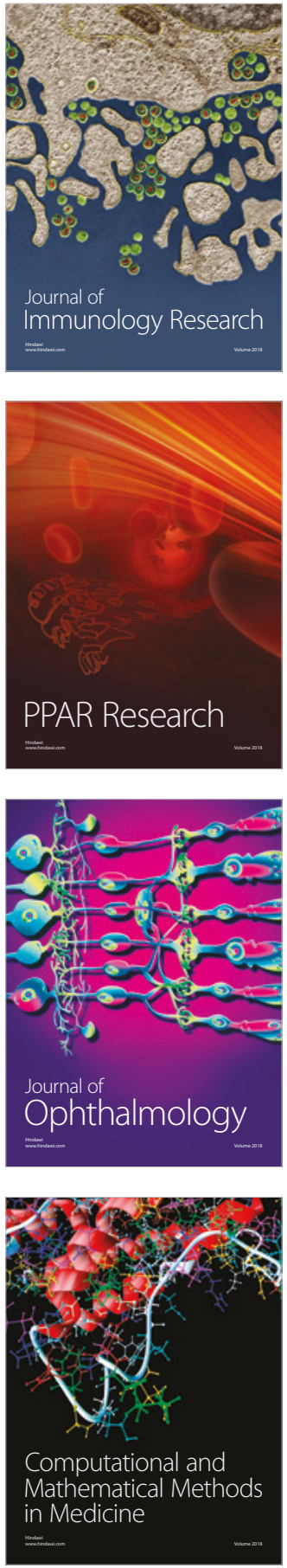

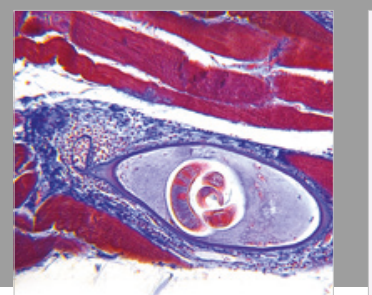

Gastroenterology Research and Practice

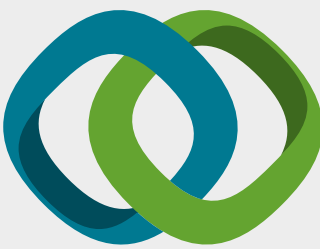

\section{Hindawi}

Submit your manuscripts at

www.hindawi.com
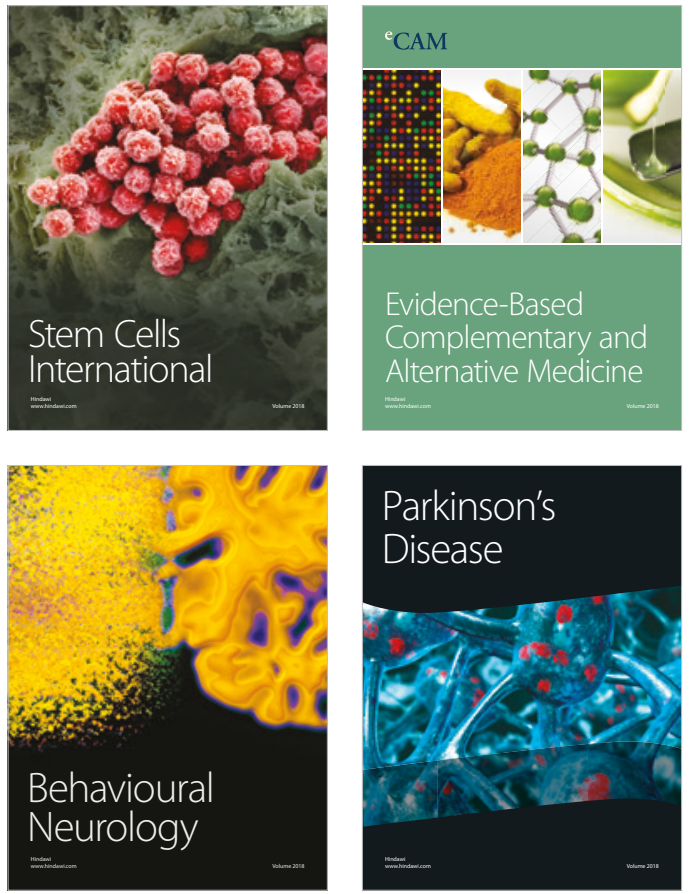

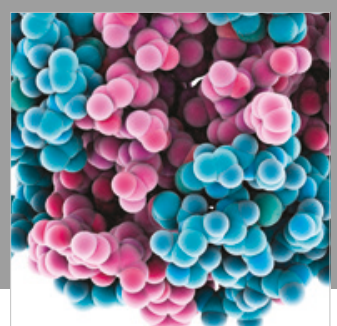

ournal of

Diabetes Research

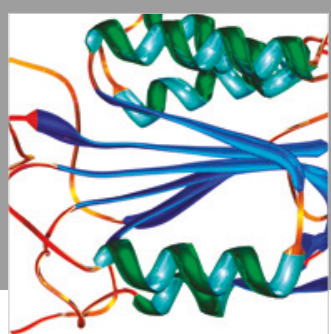

Disease Markers
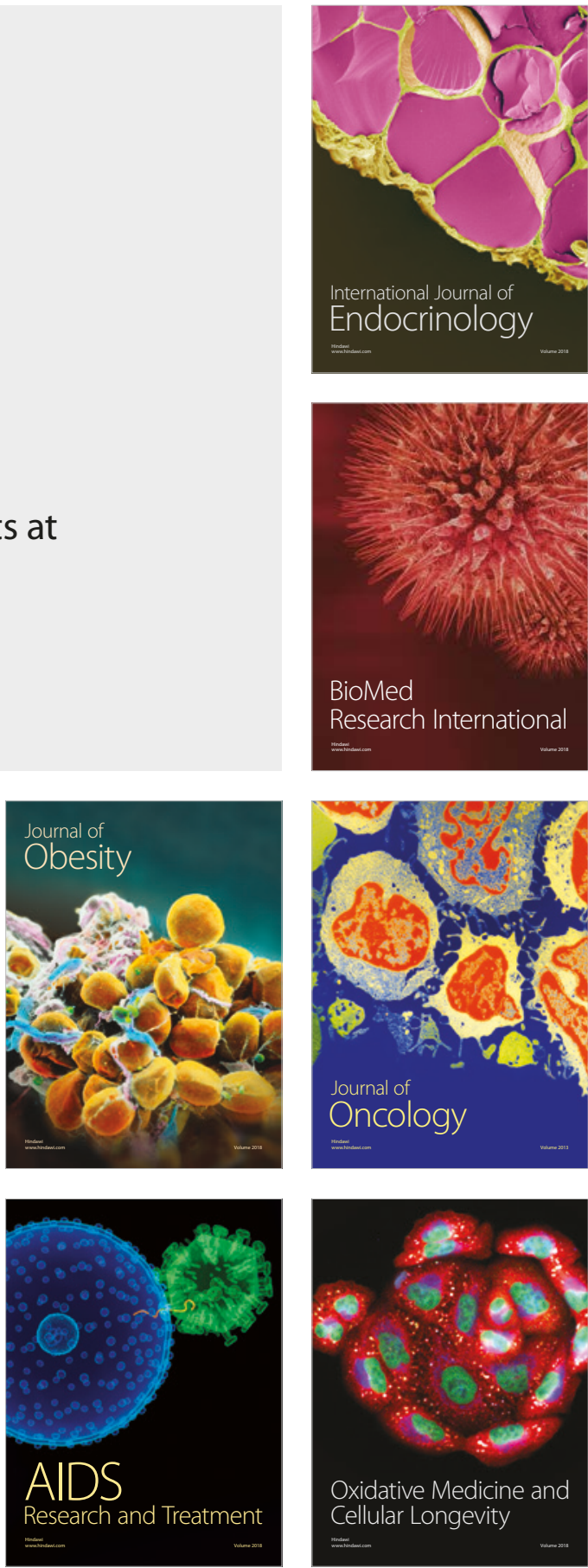